\title{
Prevalence and Anatomy of Aberrant Right Subclavian Artery Evaluated by Computed Tomographic Angiogra- phy at a Single Institution in Korea
}

\author{
Yunsuk Choi, M.D., ${ }^{1}$ Sang Bong Chung, M.D., ${ }^{1}$ Myoung Soo Kim, M.D., Ph.D., \\ Department of Neurosurgery, National Medical Center, Seoul, Korea \\ Brain Center, ${ }^{2}$ Pohang SM Christianity Hospital, Pohang, Korea
}

Objective : Aberrant right subclavian artery (ARSA) is a rare anatomical variant of the origin of the right subclavian artery. ARSA is defined as the right subclavian artery originating as the final branch of the aortic arch. The purpose of this study is to determine the prevalence and the anatomy of ARSA evaluated with computed tomography (CT) angiography.

Methods : CT angiography was performed in 3460 patients between March 1, 2014 and November 30, 2015 and the results were analyzed. The origin of the ARSA, course of the vessel, possible inadvertent ARSA puncture site during subclavian vein catheterization, Kommerell diverticula, and associated vascular anomalies were evaluated. We used the literature to review the clinical importance of ARSA.

Results : Seventeen in 3460 patients had ARSA. All ARSAs in 17 patients originated from the posterior aspect of the aortic arch and traveled along a retroesophageal course to the right thoracic outlet. All 17 ARSAs were located in the anterior portion from first to fourth thoracic vertebral bodies and were located near the right subclavian vein at the medial third of the clavicle. Only one of 17 patients presented with dysphagia.

Conclusion : It is important to be aware ARSA before surgical approaches to upper thoracic vertebrae in order to avoid complications and effect proper treatment. In patients with a known ARSA, a right transradial approach for aortography or cerebral angiography should be changed to a left radial artery or transfemoral approach.

Key Words : Aberrant subclavian artery · Computed tomography angiography · Clinical.

\section{INTRODUCTION}

Aberrant right subclavian artery (ARSA) is a rare anatomical variant of the origin of the right subclavian artery. ARSAs usually originate from the posterior aspect of the aorta and have a retroesophageal course to the right thoracic outlet. A prevalence of $0.16 \%$ to $2 \%$ of ARSA has been reported ${ }^{4,5,5,16,26,28,33)}$. Anatomical variations of the aortic arch and great vessels have historically been well documented in postmortem studies. A better understanding of this variability has been attained by

\footnotetext{
- Received : March 6, 2018 •Revised : April 26, 2018 •Accepted : April 30, 2018

-Address for reprints : Myoung Soo Kim, M.D., Ph.D.

Department of Neurosurgery, National Medical Center, 245 Eulji-ro, Jung-gu, Seoul 04564, Korea

Tel : +82-2-2260-7180, Fax : +82-2-2262-4876, E-mail : hanibalkms@hanmail.net
}

This is an Open Access article distributed under the terms of the Creative Commons Attribution Non-Commercial License (http://creativecommons.org/licenses/by-nc/4.0) which permits unrestricted non-commercial use, distribution, and reproduction in any medium, provided the original work is properly cited. 
the recent addition to the literature of relatively large studies based on radiological data from unselected living populations $^{4,5,15,16,26,27,33)}$.

We sought to determine the frequency and course of ARSA evaluated with computed tomography (CT) angiography at a single institution in Korea. We reviewed the clinical importance of ARSAs.

\section{MATERIALS AND METHODS}

After local ethics committee approval of Pohang SM Christianity Hospital was obtained for the present study (approval No. PSMCHIRB-17-116-C), images obtained by CT angiography were analyzed for the presence of ARSA. The CT angiographic studies were performed for a variety of clinical reasons, including symptoms of cerebral ischemia, hemorrhagic contusion, intracerebral hemorrhage, headache, dizziness, sensory change, and routine checkup. We excluded CT angiography performed on non-Korean patients or obtained outside hospitals. Patients younger than 18 years old were excluded. All images were evaluated by a single neurosurgeon.

CT angiography of the intracranial and extracranial vessels was performed in 3460 patients (1908 female, 1552 male; 59.10 \pm 15.02 years) between March 1, 2014 and November 30, 2015 and the results were analyzed. An Aquilion Prime 160-slice CT scanner (Toshiba Medical Systems, Tokyo, Japan) was used for 2499 patients, and an Aquilion CXL edition 128-slice CT scanner (Toshiba Medical Systems) was used in 961 patients. After the acquisition of nonenhanced CT data, contrast-enhanced CT angiography was performed. The parameters for the CT angiographic acquisition were as follows : 100 $\mathrm{kVp}, 225 \mathrm{~mA}$, field of view $220 \mathrm{~mm}$, detector collimation $80 \times$ $0.5 \mathrm{~mm}$, table speed $25.5 \mathrm{~mm} /$ rotation, gantry rotation speed $0.75 \mathrm{~s} /$ rotation, reconstructed section thickness $0.5 \mathrm{~mm}$, and reconstruction increment $0.3 \mathrm{~mm}$ for the Aquilion Prime, and $120 \mathrm{kVp}, 250 \mathrm{~mA}$, field of view $240 \mathrm{~mm}$, detector collimation $64 \times 0.5 \mathrm{~mm}$, table speed $20.5 \mathrm{~mm} /$ rotation, gantry rotation speed 0.5 s/rotation, reconstructed section thickness $0.5 \mathrm{~mm}$, and reconstruction increment $0.5 \mathrm{~mm}$ for the Aquilion CXL. The scan range extended from $2 \mathrm{~cm}$ below the aortic arch to a point $1 \mathrm{~cm}$ above the level of the lateral ventricles.

The results of CT angiography were analyzed using the following methods. A total of $100 \mathrm{~mL}$ of iopamidol (Pamiray
370; Dongkook Pharmaceuticals, Seoul, Korea) was administered intravenously using a power injector at $4.0 \mathrm{~mL} / \mathrm{s}$ via an 18-gauge catheter positioned in a peripheral vein, and the scan delay adapted individually using a bolus-tracking technique. For the bolus tracking, first, a single nonenhanced low-dose scan at the level of the upper neck was obtained. With the start of contrast material administration, repeated low-dose monitoring scans were obtained every second. When the contrast medium was first seen in the common carotid artery (CCA), the CT angiography was triggered automatically without any delay. The data were transferred to a personal computer and three-dimensional (3D) reconstructions of the images were obtained using commercially available software (Vitrea 2; Vital Images, Minnetonka, MN, USA). From these data, 3D CT angiography images were reconstructed using a volume-rendering technique. A series of 17 projection images at every $20^{\circ}$ around the cephalocaudal axis were generated and then transferred to a picture archiving and communication system.

The origin of the ARSA, course of the vessel, possible inadvertent ARSA puncture site during subclavian vein catheterization, Kommerell diverticula, and associated vascular anomalies were evaluated. A Kommerell diverticulum in ARSA was defined as a widening of the base of the subclavian artery to $>1.5$ times the size of the distal subclavian artery ${ }^{12)}$.

\section{RESULTS}

Seventeen in 3460 patients had ARSA. All ARSAs in 17 patients $(17 / 3460=0.49 \%, 13$ female, four male; age range, $22-78$ years) originated from the posterior aspect of the aortic arch and traveled along a retroesophageal course to the right thoracic outlet. Characteristics of all 17 ARSAs are presented in Table 1. ARSAs had two different origins from the aortic arch. Two ARSAs (cases 6 and 15) had an inferoposterior origin from the aortic arch (Fig. 1); the other 15 ARSAs had an origin from a superoposterior direction (Fig. 2). Subsequently, the ARSAs had three different courses between the thoracic vertebra body and esophagus. One ARSA (case 7) had a horizontal course anterior to the third thoracic vertebral body. Two ARSAs (cases 8 and 15) had a horizontal course anterior to the vertebrae and ascended to the right side of the vertebrae (Fig. 1). The other 14 ARSAs had an oblique course in the anterior 
Table 1. Characteristics of all 17 ARSAs in patients in the present study

\begin{tabular}{|c|c|c|c|c|c|c|c|}
\hline Number & Age & Sex & ARSA origin* & $\begin{array}{c}\text { Vertebral body } \\
\text { level }^{\dagger}\end{array}$ & $\begin{array}{l}\text { Kommerell } \\
\text { diverticulum }\end{array}$ & $\begin{array}{l}\text { Origin of both } \\
\text { CCAs }\end{array}$ & Associated vascular anomaly \\
\hline 1 & 22 & $F$ & Superoposterior & $\mathrm{T} 2-\mathrm{T} 4$ & Negative & Separate & \\
\hline 2 & 29 & $\mathrm{~F}$ & Superoposterior & $\mathrm{T} 2-\mathrm{T} 4$ & Positive & Common stem & Left VA originated from aorta \\
\hline 3 & 36 & $\mathrm{~F}$ & Superoposterior & $\mathrm{T} 2-\mathrm{T} 3$ & Positive & Separate & \\
\hline 4 & 43 & F & Superoposterior & $\mathrm{T} 2-\mathrm{T} 3$ & Negative & Common stem & \\
\hline 5 & 45 & $\mathrm{~F}$ & Superoposterior & $\mathrm{T} 2-\mathrm{T} 3$ & Negative & Common stem & \\
\hline 6 & 48 & $F$ & Inferoposterior & $\mathrm{T} 2-\mathrm{T} 4$ & Positive & Common stem & Accessory MCA \\
\hline 7 & 56 & $M$ & Superoposterior & $\mathrm{T} 2$ & Negative & Common stem & \\
\hline 8 & 58 & $\mathrm{~F}$ & Superoposterior & $\mathrm{T} 2-\mathrm{T} 3$ & Negative & Separate & \\
\hline 9 & 59 & $M$ & Superoposterior & $\mathrm{T} 2-\mathrm{T} 3$ & Positive & Common stem & \\
\hline 10 & 60 & F & Superoposterior & $\mathrm{T} 1-\mathrm{T} 2$ & Negative & Separate & \\
\hline 11 & 60 & $\mathrm{~F}$ & Superoposterior & $\mathrm{T} 2-\mathrm{T} 3$ & Positive & Common stem & Fenestration of BA \\
\hline 12 & 71 & $M$ & Superoposterior & $\mathrm{T} 2$ & Negative & Separate & $\begin{array}{l}\text { C2 segmental artery }{ }^{\ddagger} \text {, fenestration of } \\
\text { A1 }\end{array}$ \\
\hline 13 & 72 & $F$ & Superoposterior & $\mathrm{T} 1-\mathrm{T} 2$ & Positive & Common stem & \\
\hline 14 & 72 & $M$ & Superoposterior & $\mathrm{T} 2-\mathrm{T} 3$ & Positive & Common stem & \\
\hline 15 & 72 & $\mathrm{~F}$ & Inferoposterior & $\mathrm{T} 2-\mathrm{T} 4$ & Positive & Separate & $\begin{array}{l}\text { Fenestration of BA, Left VA originated } \\
\text { from aorta, } C 2 \text { segmental artery }{ }^{\ddagger}\end{array}$ \\
\hline 16 & 75 & $\mathrm{~F}$ & Superoposterior & $\mathrm{T} 2-\mathrm{T} 3$ & Positive & Common stem & \\
\hline 17 & 78 & $\mathrm{~F}$ & Superoposterior & $\mathrm{T} 1-\mathrm{T} 2$ & Negative & Separate & Fenestration of $A 1$ \\
\hline
\end{tabular}

*ARSA origin : direction of ARSA origin from aortic arch. ${ }^{\dagger}$ Vertebral body level : thoracic vertebral body levels which the ARSA coursed in the anterior and lateral portion of thoracic vertebral bodies. ${ }^{\ddagger}$ Previously described as persistent first intersegmental artery. ARSA : aberrant right subclavian artery, CCA : common carotid artery, F : female, VA : vertebral artery, MCA : middle cerebral artery, M : male, BA : basilar artery, A1 : precommunicating segment of anterior cerebral artery
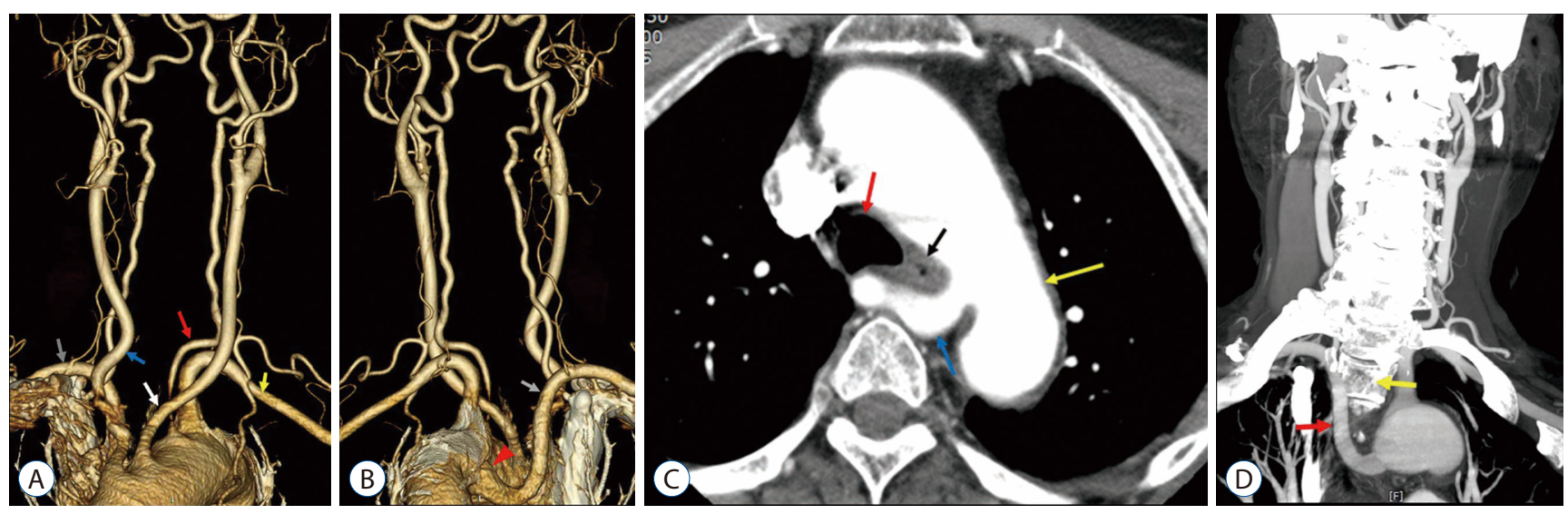

Fig. 1. A 72-year-old woman with an aberrant right subclavian artery (ARSA) with an inferoposterior origin (case 15). A : Anterior view of the aortic arch branch showing separate origin of both common carotid arteries (CCAs) and the left vertebral artery (VA) from the aorta (red arrow, left VA originating from the aortic arch; yellow arrow, left subclavian artery; white arrow, left CCA; blue arrow, right CCA; gray arrow, right subclavian artery). B : Posterior view of the aortic arch branch showing the ARSA (gray arrow, ARSA; red arrowhead, Kommerell diverticulum). C: Axial image showing the ARSA located anterior to the thoracic vertebrae (yellow arrow, aorta; red arrow, trachea; black arrow, esophagus; blue arrow, ARSA). D : Coronal image showing the ARSA with a horizontal course anterior to the vertebral body and an ascending course to the right side of the vertebral body (red arrow, ARSA; yellow arrow, second thoracic vertebral body). 

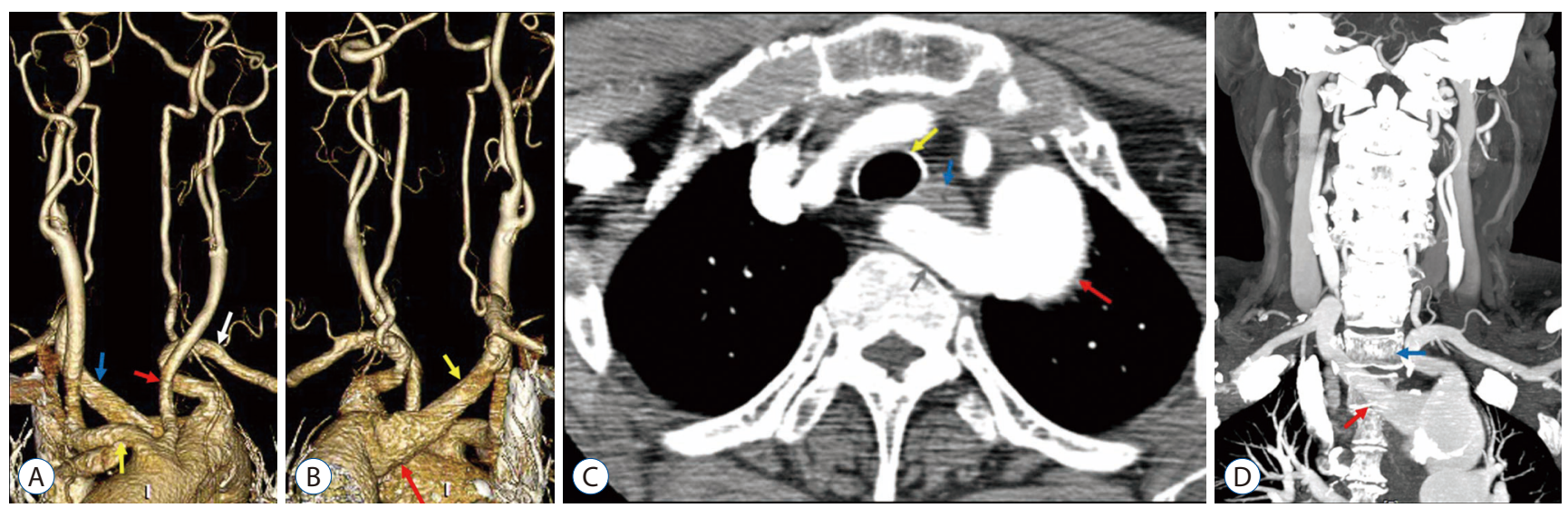

Fig. 2. A 60-year-old woman with an aberrant right subclavian artery (ARSA) with a superoposterior origin (case 11). A : Anterior view of the aortic arch branch showing the common origin of both common carotid arteries (CCAs) (red arrow, left CCA; yellow arrow, right CCA; white arrow, left subclavian artery; blue arrow, ARSA). B : Posterior view of the aortic arch branch showing the ARSA (yellow arrow, ARSA; red arrow, Kommerell diverticulum). C : Axial image showing the ARSA located anterior to the thoracic vertebrae (yellow arrow, trachea; red arrow, aorta; blue arrow, esophagus; gray arrow, ARSA). D : Coronal image showing the ARSA with an oblique course in the anterior portion of the thoracic vertebrae (red arrow, ARSA; blue arrow, first thoracic vertebral body).
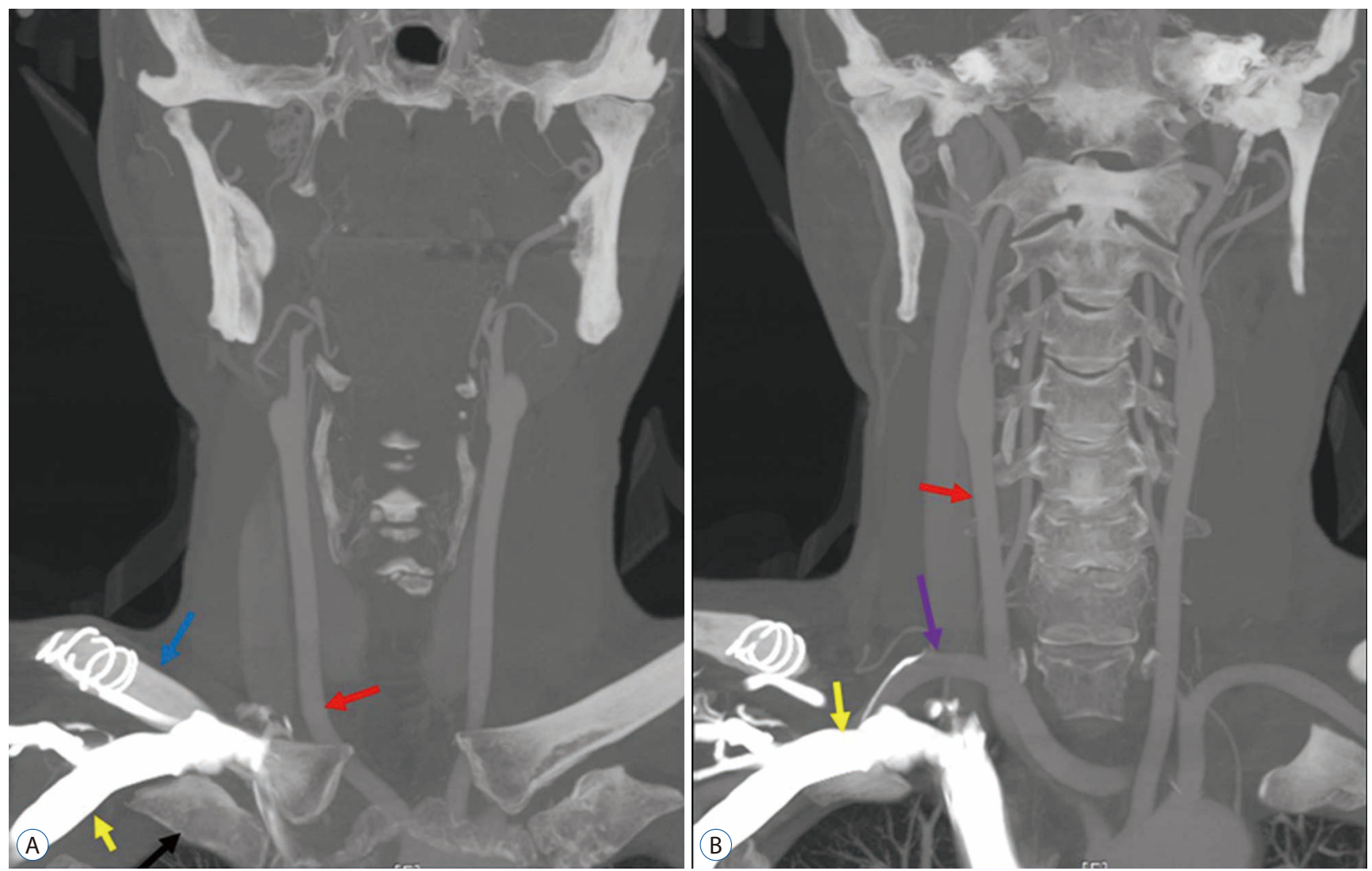

Fig. 3. A 56-year-old man with an aberrant right subclavian artery (ARSA) (case 7). A : Coronal image showing the subclavian vein (yellow arrow) located between the clavicle (blue arrow) and first rib (black arrow). Red arrow indicates right common carotid artery. B : Coronal image showing close proximity of the ARSA (violet arrow) and right subclavian vein (yellow arrow). Red arrow indicates right common carotid artery. 
thoracic vertebrae (one thoracic vertebral level in one patient [case 12], two thoracic vertebral levels in 10, and three thoracic vertebral levels in three [cases 1, 2, and 6]) (Fig. 2). Nine of 17 cases were confirmed to have a Kommerell diverticulum and only one (case 10) of 17 patients presented with dysphagia.

All 17 ARSAs were posterior to the right subclavian vein. Possible inadvertent ARSA puncture sites during subclavian vein catheterization in all 17 ARSAs were in the medial third of the clavicle. In this area, the ARSA and subclavian vein were located in close proximity (Fig. 3).

Associated vascular anomalies included two aortic origins of the left vertebral artery, one accessory middle cerebral artery, two C2 segmental arteries, two fenestrations of the basilar artery, and two fenestrations of A1. Both CCA had a common stem from the aortic arch in 10 cases and a separate origin from aortic arch in seven cases.

\section{DISCUSSION}

\section{Embryogenic mechanism of ARSA}

During human embryogenesis, the aortic arches appear in the 4 th week of fetal development. Normally, six aortic arch pairs appear. In the normal development of the right subclavian artery, the proximal part of the artery is formed from the most caudal portion of the right dorsal aorta, while the distal portion is formed by the 7 th right intersegmental artery ${ }^{20)}$. Departure from the normal embryologic developmental pattern of the primitive aortas and aortic arches results in the formation of an ARSA. The 4th vascular arch involutes along with the right dorsal aorta, while the 7th intersegmental artery remains attached to the descending aorta. This persistent intersegmental artery becomes an ARSA formed from the distal aortic root and the 7 th intersegmental artery ${ }^{20,28)}$.

Shortening of the left distal aortic root brings the origin of the ARSA just distal to the normal left subclavian artery ${ }^{22)}$. Because the persisting right aortic arch forms the root of the ARSA, the artery often has a broad base, which is referred to as a Kommerell diverticulum ${ }^{19)}$. The stem of the anomalous subclavian artery is derived from part of the right dorsal aorta; this explains the retroesophageal course that this artery takes as it passes to the right upper $\operatorname{limb} b^{30,32)}$.

Ten of 17 patients with ARSA in this study had common stem of both CCAs. This vessel anomaly is similar with bo- vine type aortic arch. Bovine type aortic arch refers to both a shared origin of the left CCA and innominate artery, and an origin of the left CCA from the innominate artery ${ }^{21)}$. A bovine arch is thought to result from slow growth or regression of the ventral aortic roots between the 3rd and 4th aortic arches, resulting in fusion of the left CCA and innominate artery ${ }^{1,21,25,29)}$. The involution of the 4th aortic arch and the right dorsal aorta result in ARSA. Fourth aortic arch and right dorsal aorta are located in close position from ventral aortic roots between the 3rd and 4th aortic arches. In our opinion, slow growth or regression of the ventral aortic roots between the 3 rd and 4 th aortic arches in ARSA development may result to common stem of both CCAs. The embryologic development of an ARSA and common stem of both CCAs is shown in Fig. 4.

\section{Clinical significance}

In 1794, Bayford ${ }^{3)}$ described a 62-year-old woman who died after years of dysphagia. On autopsy, an ARSA compressing the esophagus was identified. Previous studies have reported a prevalence of $0.16 \%$ to $2 \%$ of $\mathrm{ARSA}^{4,5,15,16,25,28,33)}$; the rate was $0.49 \%(17 / 3460)$ in the present study. ARSA is a rare anatomical variant of the origin of the right subclavian artery. ARSA originates from the left half of the body, and in its course to the right arm, it usually (85\%) crosses the midline behind the esophagus, and is thus known as retroesophageal ARSA ${ }^{2,28)}$. Furthermore, it may run between the trachea and the esophagus or in front of the trachea ${ }^{6}$. Based on autopsy studies and retrospective analysis of patients' symptoms during life, Janssen et al. ${ }^{17)}$ found that a substantial proportion (60-70\%) of patients remain symptom-free during their lifetime. Coughing, dysphagia, thoracic pain, and Horner syndrome may develop during aging. It is unknown why dysphagia may develop in the older patient. Various mechanisms are proposed : 1) increased rigidity of the vessel wall or the esophagus itself; 2) aneurysm formation, especially in the presence of a Kommerell diverticulum; and 3) elongation of the aorta ${ }^{18)}$. Only one of the 17 patients in the present study presented with dysphagia, which improved after conservative treatment.

Sixty percent of ARSA are associated with a Kommerell diverticulum. This dilatation at the origin of the ARSA is the embryologic remnant ${ }^{19)}$. In the present study, nine of 17 cases had a Kommerell diverticulum.

If the ARSA comes in contact with the trachea, this may cause dyspnea, whereas if it is found in front of the trachea, 


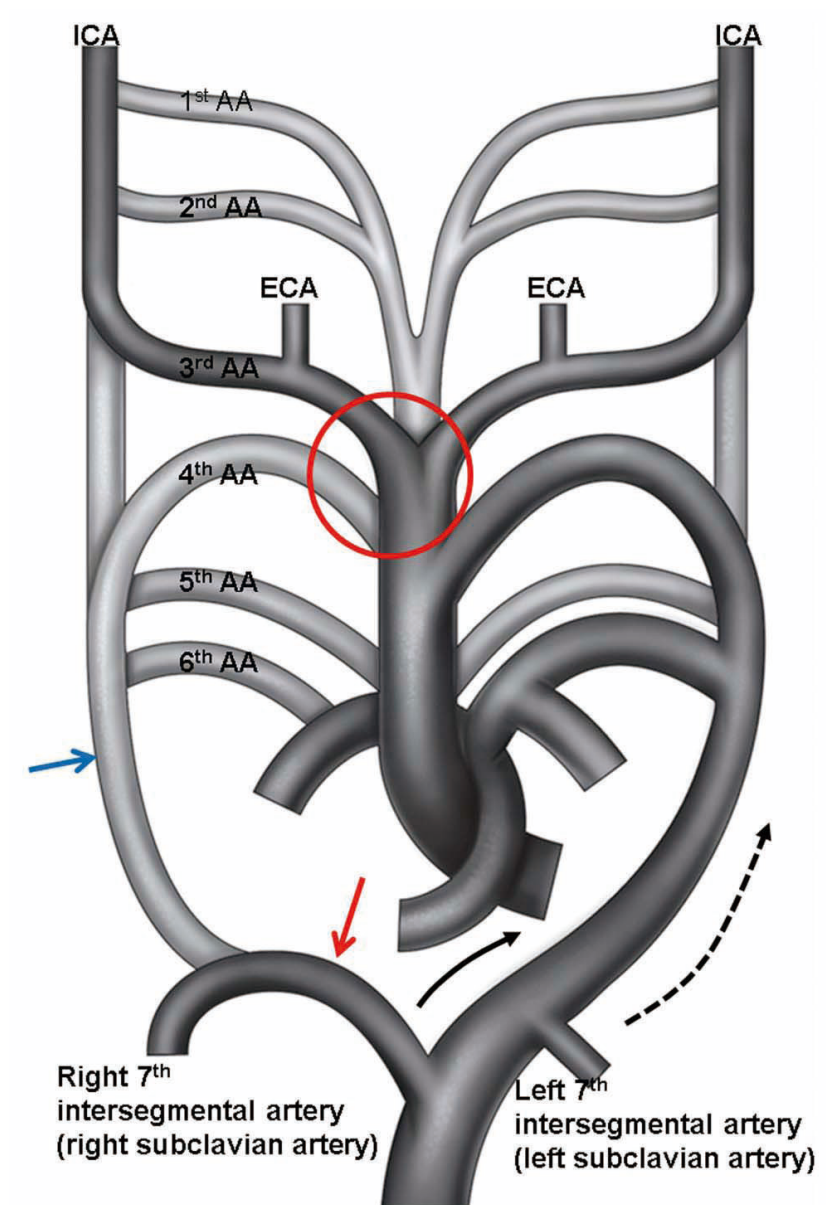

Fig. 4. Abnormal embryonic development of the great vessels leading to the formation of an ARSA and common stem of both CCAs. Embryonic development of the AA takes place during the 4th and 8th week of fetal life. Normal embryonic development of the $A A$ and great vessels begins as six paired AAs. The 1 st and 2 nd AAs regress. The paired $3 \mathrm{rd}$ arches form the 1st part of the ICA bilaterally. The proximal right 4th arch persists as the right subclavian artery at the origin of the internal mammary artery, whereas the distal right 4 th arch regresses. The left 4 th arch forms the anatomical basis of the subsequent fully formed AA. The 5th arch has not been formed completely. In abnormal embryonic development, involution of the right 4th AA and proximal right dorsal aorta leaves the right 7th intersegmental artery to arise from the left dorsal aorta, resulting in an ARSA. With further development, differential growth shifts the origin of the ARSA and the left subclavian artery cranially. Regression of the ventral aortic roots between the 3rd and 4th AAs in red circle of this figure result to common stem of both CCAs. Black half tone vessel indicates fully developed vessel in adult. Gray half tone vessel indicates obliterated vessel in development. Blue arrow indicates abnormally disappeared proximal right dorsal aorta, red arrow is persistent distal right dorsal aorta, and black and black dotted arrows are growth direction of both 7th intersegmental arteries. ICA : internal carotid artery, AA : aortic arch, ECA : external carotid artery, ARSA : aberrant right subclavian artery, CCA : common carotid artery. this may cause catastrophic complications during tracheostomy ${ }^{6}$. Desvant et al. ${ }^{7)}$ reported that aneurysmal disruption of a retroesophageal ARSA in patients with spinal deformities should be kept in mind as a potential cause of tracheostomy bleeding in patients requiring long-term follow-up.

A fistula between the esophagus and an ARSA remains an exceptional event that has mostly been described in association with prolonged nasogastric intubation ${ }^{13,24)}$. The abnormal anatomic proximity to the esophagus or trachea likely renders the ARSA vulnerable to extrinsic compression and pressure necrosis by indwelling nasogastric or endotracheal tubes ${ }^{9,10)}$. The sequence of events may involve occlusion and thrombosis of the vasa vasorum, leading to vessel wall infarction and eventual wall dissolution. Moreover, ischemia and bacterial invasion of the vessel wall have been suggested as important etiologic factors ${ }^{8)}$. Diagnostic difficulty in patients with massive hemorrhage secondary to a fistula between an ARSA and the esophagus results in the high mortality of patients with this complication $^{23)}$. To avoid the devastating consequences of an ARSA-esophageal fistula, we reiterate the warning of Miller et al. ${ }^{24)}$ to avoid prolonged nasogastric and endotracheal intubation in patients known to have an ARSA or other vascular ring anomaly; we recommend early gastrostomy, early tracheostomy, or early removal of nasogastric intubation or tracheal intubation.

ARSAs have a course close to the thoracic vertebrae. When performing an anterior approach to the upper thoracic vertebrae, the area at the level above the aortic arch in the space between the esophagus and the spine is usually regarded as a safe area for dissection of upper thoracic vertebrae. If surgeons do not consider the ARSA, pedicle screw insertion into a thoracic vertebra via a posterior approach or thoracic vertebral body removal using an anterior approach may result in uncontrollable bleeding. Thorpe et al. ${ }^{31)}$ reported that ARSA bleeding in a case of debridement of T2 for osteomyelitis resulted in a complication and eventual mortality. All 17 ARSAs in the present study were located in the anterior region of the first to fourth thoracic bodies. When planning an operative approach to the upper thoracic region, surgeons should be aware of anatomic variants of ARSAs ${ }^{31}$.

A transradial approach has been used in diagnostic angiography and interventional treatment. A diagnosis of ARSA should be suspected if the guide wire repeatedly enters the descending aorta from the right subclavian artery rather than 
the ascending aorta during aortography via the right radial artery. In this case, catheterization of the ascending aorta may be difficult or even impossible because of the angular course of the ARSA to the ascending aorta ${ }^{18)}$ as in this study. Because the success rate of the right transradial approach in the setting of an ARSA is only $60 \%$, with an additional potential risk of dissection as in the patient reported by Huang et al. ${ }^{11}$, arch anomaly should be considered and included in preintervention planning. In this case, we recommend angiography via the left radial artery or using a transfemoral approach.

Jahnke et al. ${ }^{14)}$ reported that inadvertent puncture of an ARSA during right subclavian vein catheterization may lead to a potentially fatal complication. Several different skin entry sites are described in the literature. Some practitioners consider that the preferred entry site is $1 \mathrm{~cm}$ caudal to the junction of the medial and middle thirds of the clavicle. Other practitioners prefer to enter the skin inferior to the clavicle at the deltopectoral groove, or the point just lateral to the midclavicular line along the inferior surface of the clavicle. We consider that the close proximity of the subclavian vein and ARSA at the medial third of clavicle may risk unintentional puncture of the ARSA during subclavian catheterization. Ultrasoundguided puncture for central venous lines should be used wherever possible, and is especially encouraged when an ARSA has been detected.

\section{CONCLUSION}

ARSA is rare variation of the branch from the aortic arch. Clinicians should be aware of the anatomy and clinical importance of an ARSA. In particular, to avoid long-term use of a nasogastric tube and the devastating consequences of an ARSA-esophageal fistula, it is important to be aware ARSA, as well as preoperative identification of ARSA before surgical approaches to upper thoracic vertebrae in order to avoid complications and effect proper treatment. In patients with a known ARSA, a right transradial approach for aortography or cerebral angiography should be changed to a left radial artery or transfemoral approach.

\section{CONFLICTS OF INTEREST}

No potential conflict of interest relevant to this article was reported.

\section{INFORMED CONSENT}

This type of study does not require informed consent.

\section{- Acknowledgements}

This work was supported by research grant from a National Medical Center and Pohang SM Christianity Hospital.

\section{References}

1. Babu CS, Sharma $V$ : Two common trunks arising from arch of aorta: case report and literature review of a very rare variation. J Clin Diagn Res 9 : AD05-AD07, 2015

2. Backer $\mathrm{CL}$, Ilbawi $\mathrm{MN}$, Idriss FS, DeLeon SY : Vascular anomalies causing tracheoesophageal compression. Review of experience in children. J Thorac Cardiovasc Surg 97 : 725-731, 1989

3. Bayford $D:$ An account of singular case of obstructed deglutition. Mem Med Soc Lond 2 : 275-286, 1794

4. Berko NS, Jain VR, Godelman A, Stein EG, Ghosh S, Haramati LB : Variants and anomalies of thoracic vasculature on computed tomographic angiography in adults. J Comput Assist Tomogr 33 : 523-528, 2009

5. Celikyay ZR, Koner AE, Celikyay F, Denız C, Acu B, Firat MM : Frequency and imaging findings of variations in human aortic arch anatomy based on multidetector computed tomography data. Clin Imaging 37 : 10111019, 2013

6. Chadha NK, Chiti-Batelli $S$ : Tracheostomy reveals a rare aberrant right subclavian artery; a case report. BMC Ear Nose Throat Disord $4: 1$, 2004

7. Desvant C, Chevalier D, Mortuaire G : Tracheotomy bleeding from an unusual tracheo-arterial fistula: involvement of an aberrant right subclavian artery. J Laryngol Otol 124 : 1333-1336, 2010

8. Gable DS, Stoddard LD : Acute bacterial aortitis resulting in an aortoesophageal fistula. A fatal complication of untreated esophageal carcinoma. Pathol Res Pract 184 : 318-324, 1989

9. Heck HA Jr, Moore HV, Lutin WA, Leatherbury L, Truemper EJ, Steinhart $C M$, et al. : Esophageal-aortic erosion associated with double aortic arch and tracheomalacia. Experience with 2 infants. Tex Heart Inst J 20: 126-129, 1993

10. Hosn MA, Haddad F, El-Merhi F, Safadi B, Hallal A : Repair of an aberrant subclavian arterioesophageal fistula following esophageal stent 
placement. World J Gastrointest Surg 6 : 117-121, 2014

11. Huang IL, Hwang HR, Li SC, Chen CK, Liu CP, Wu MT : Dissection of arteria lusoria by transradial coronary catheterization: a rare complication evaluated by multidetector CT. J Chin Med Assoc 72 : 379-381, 2009

12. Ichikawa T, Koizumi J, Tanno K, Okochi T, Nomura T, Shimura S, et al. : Kommerell diverticulum in adults: evaluation of routine CT examinations. Tokai J Exp Clin Med 41 : 65-69, 2016

13. Inman JC, Kim P, McHugh R : Retroesophageal subclavian artery-esophageal fistula: a rare complication of a salivary bypass tube. Head Neck 30 : 1120-1123, 2008

14. Jahnke T, Schaefer PJ, Heller M, Mueller-Huelsbeck S : Interventional management of massive hemothorax due to inadvertent puncture of an aberrant right subclavian artery. Cardiovasc Intervent Radiol 31 : Suppl 2:S124-S127, 2008

15. Jakanani GC, Adair W : Frequency of variations in aortic arch anatomy depicted on multidetector CT. Clin Radiol 65 : 481-487, 2010

16. Jalali Kondori B, Asadi MH, Rahimian E, Tahsini MR : Anatomical variations in aortic arch branching pattern. Arch Iran Med 19 : 72-74, 2016

17. Janssen M, Baggen MG, Veen HF, Smout AJ, Bekkers JA, JG, et al. : Dysphagia lusoria: clinical aspects, manometric findings, diagnosis, and therapy. Am J Gastroenterol 95 : 1411-1416, 2000

18. Jiang $X H$, Zhu $X Y$ : Abnormal origin of the right subclavian artery: a case report. Chin Med J (Engl) 130 : 1508-1509, 2017

19. Kiernan PD, Dearani J, Byrne WD, Ehrlich T, Carter W, Krasicky G, et al. : Aneurysm of an aberrant right subclavian artery: case report and review of the literature. Mayo Clin Proc 68 : 468-474, 1993

20. Levitt B, Richter JE : Dysphagia lusoria: a comprehensive review. Dis Esophagus 20 : 455-460, 2007

21. Malone CD, Urbania TH, Crook SE, Hope MD : Bovine aortic arch: a novel association with thoracic aortic dilation. Clin Radiol 67 : 28-31, 2012

22. Maranillo E, Vazquez T, Quer $M$, Niedenführ MR, Leon X, Viejo $F$, et al. : Potential structures that could be confused with a nonrecurrent inferior laryngeal nerve: an anatomic study. Laryngoscope 118 : 56-60, 2008

23. Merchant FJ, Nichols RL, Bombeck CT : Unusual complication of naso- gastric esophageal intubation-erosion into an aberrant right subclavian artery. J Cardiovasc Surg (Torino) 18 : 147-150, 1977

24. Miller RG, Robie DK, Davis SL, Cooley DA, Klish WJ, Skolkin MD, et al. : Survival after aberrant right subclavian artery-esophageal fistula: case report and literature review. J Vasc Surg 24 : 271-275, 1996

25. Moorehead PA, Kim AH, Miller CP, Kashyap TV, Kendrick DE, Kashyap VS : Prevalence of bovine aortic arch configuration in adult patients with and without thoracic aortic pathology. Ann Vasc Surg 30 : 132-137, 2016

26. Müller M, Schmitz BL, Pauls S, Schick M, Röhrer S, Kapapa T, et al. : Variations of the aortic arch - a study on the most common branching patterns. Acta Radiol 52 : 738-742, 2011

27. Natsis K, Didagelos M, Manoli SM, Papathanasiou E, Sofidis G, Anastasopoulos $\mathrm{N}$ : A bicarotid trunk in association with an aberrant right subclavian artery. Report of two cases, clinical impact, and review of the literature. Folia Morphol (Warsz) 70 : 68-73, 2011

28. Natsis KI, Tsitouridis I A, Didagelos MV, Fillipidis AA, Vlasis KG, Tsikaras $\mathrm{PD}$ : Anatomical variations in the branches of the human aortic arch in 633 angiographies: clinical significance and literature review. Surg Radiol Anat $31:$ 319-323, 2009

29. Nelson ML, Sparks CD : Unusual aortic arch variation: distal origin of common carotid arteries. Clin Anat 14 : 62-65, 2001

30. Sukumaran TT, Pillay M, Gopalakrishnan A : An anomalous right subclavian artery with a retrotracheal course: a case report. J Clin Diagn Res 9 : AD01-AD02, 2015

31. Thorpe SW, Hohl JB, Gilbert S, Tannoury CA, Lee JY : Aberrant right subclavian artery encountered during debridement of $\mathrm{T} 2$ osteomyelitis and associated phlegmon. Spine J 11 : e6-e10, 2011

32. Tubbs RS, Oakes WJ, Salter EG, Zehren SJ : Retroesophageal right subclavian artery with persistent ductus arteriosus. Anat Sci Int 79 : 98 100,2004

33. Vučurević G, Marinković S, Puškaš L, Kovačević I, Tanasković S, Radak D, et al. : Anatomy and radiology of the variations of aortic arch branches in 1,266 patients. Folia Morphol (Warsz) 72 : 113-122, 2013 